\title{
Communication
}

Subscriber access provided by Nottingham Trent University

\section{A Triazine Functionalized Porous Covalent Organic Framework for Photo-organocatalytic E-Z Isomerization of Olefins}

\author{
Mohitosh Bhadra, Sharath Kandambeth, Manoj K. Sahoo, Matthew \\ Addicoat, Dr. Ekambaram Balaraman, and Rahul Banerjee
}

J. Am. Chem. Soc., Just Accepted Manuscript • DOI: 10.1021/jacs.9b01891 • Publication Date (Web): 04 Apr 2019

Downloaded from http://pubs.acs.org on April 7, 2019

\section{Just Accepted}

"Just Accepted" manuscripts have been peer-reviewed and accepted for publication. They are posted online prior to technical editing, formatting for publication and author proofing. The American Chemical Society provides "Just Accepted" as a service to the research community to expedite the dissemination of scientific material as soon as possible after acceptance. "Just Accepted" manuscripts appear in full in PDF format accompanied by an HTML abstract. "Just Accepted" manuscripts have been fully peer reviewed, but should not be considered the official version of record. They are citable by the Digital Object Identifier (DOI®). "Just Accepted" is an optional service offered to authors. Therefore, the "Just Accepted" Web site may not include all articles that will be published in the journal. After a manuscript is technically edited and formatted, it will be removed from the "Just Accepted" Web site and published as an ASAP article. Note that technical editing may introduce minor changes to the manuscript text and/or graphics which could affect content, and all legal disclaimers and ethical guidelines that apply to the journal pertain. ACS cannot be held responsible for errors or consequences arising from the use of information contained in these "Just Accepted" manuscripts. 


\title{
A Triazine Functionalized Porous Covalent Organic Framework for Photo-organocatalytic $E$-Z Isomerization of Olefins
}

\author{
Mohitosh Bhadra, ${ }^{\text {a,b }}$ Sharath Kandambeth, ${ }^{\mathrm{b}}$ Manoj K. Sahoo, ${ }^{\mathrm{a}, \mathrm{b}}$ Matthew Addicoat, ${ }^{\mathrm{d}}$ Ekambaram Bala- \\ raman $^{* a, b}$ and Rahul Banerjee ${ }^{* c}$ \\ ${ }^{a}$ Academy of Scientific and Innovative Research (AcSIR), CSIR-National Chemical Laboratory, Dr. Homi Bhabha Road, \\ Pune-411008, India \\ ${ }^{b}$ Physical/Materials and Organic Chemistry Division, CSIR-National Chemical Laboratory, Dr. Homi Bhabha Road, Pune- \\ 411008, India \\ ${ }^{c}$ Department of Chemical Sciences, Indian Institute of Science Education and Research (IISER) Kolkata, Mohanpur Campus, \\ Mohanpur, 741246, India \\ ${ }^{\mathrm{d} S}$ School of Science and Technology, Nottingham Trent University, Clifton Lane, NG11 8NS Nottingham, United Kingdom
}

Supporting Information Placeholder

\begin{abstract}
Visible light-mediated photocatalytic organic transformation has drawn significant attention as an alternative process for replacing thermal reactions. Although precious metal/organic dyes based homogeneous photocatalysts have been developed, their toxic and non-reusable nature makes them inappropriate for large-scale production. Therefore, we have synthesized a triazine and a keto functionalized non-metal based covalent organic framework (TpTt) for heterogeneous photocatalysis. As the catalyst shows significant absorption of visible light, it has been applied for the photocatalytic uphill conversion of transstilbene to cis-stilbene in the presence of blue LEDs with broad substrates scope via an energy transfer process.
\end{abstract}

\footnotetext{
Alkene/olefin functional groups are essential organic building units in many synthetic polymers and drugs. ${ }^{1-c}$ Stereoselective synthesis of olefins is important due to their large scope of applications in synthesizing anticancer drugs, ${ }^{\text {1d,e }}$ scintillators, ${ }^{2}$ chromatic lasers ${ }^{3}$ and industrial dyes. ${ }^{4,5}$ However, most of the methods to synthesize alkenes lead to the formation of the thermodynamically more stable trans $(E)$ form while the direct synthesis of the energetically less stable cis (Z) form is remarkably challenging and has been restricted to a limited number of available synthetic methods. ${ }^{6}$ Most of these synthetic methods lack high stereoselectivity and often require high energy and expensive homogeneous metal catalysts. ${ }^{7}$ The probable solution for an economic and stereoselective synthesis of the $Z$ isomer should be to follow the conventional photo-assisted $E$ to $Z$ isomerization strategy. However, most of the common olefin compounds lack absorption in the visible range. Thus, the $E$ to $Z$ transformation can happen only by a high energy UV radiation approach, which is neither a green, nor a safe process. ${ }^{8}$ The alternative solution for this problem would be to use more abundant visible light to perform this $E$ to $Z$ isomerization of olefins with the help of a photocatalyst. However, designing such catalyst is a challenging task as the ideal photocatalyst needs to feature high photochemical stability, optimum band gap, absorption maxima in the visible range, and a long-lived excited state. ${ }^{9}$ In the literature, photocatalysts such as metal polypyridyl complexes, ${ }^{10}$ organic dyes such as riboflavin, ${ }^{11}$ and aromatic keto
}

compounds ${ }^{12}$ have been used for the $E$ to $Z$ isomerization of alkenes. However, the recyclability of these expensive photocatalysts becomes a key issue due to their homogenous nature and low photochemical stability. Hence, there is a high demand for developing a novel heterogeneous, chemically stable photocatalyst for the economic and energy efficient $E$ to $Z$ conversion of alkenes.

Covalent Organic Frameworks (COFs), a novel class of porous crystalline polymers, have recently emerged as heterogeneous catalysts for various organic transformations ${ }^{13} \mathrm{COFs}$, because of their highly ordered, predesignable and functionalizable porous structures, allow precise integration of catalytic centers in the framework matrix in a well-defined manner. Keeping all these features in perspective, we have successfully synthesized a novel heterogeneous $\mathrm{COF}$ based porous photocatalyst for the $E$ to $Z$ isomerization of alkenes. The photocatalytic COF is constructed from two distinct photoactive building blocks (triazine and $\beta$ ketoenamine) having unique photo-sensitizing properties. The triazine core should catalyze $E$ to $Z$ photo-isomerization of an alkene by facilitating strong $\pi-\pi$ interaction with the $E$ alkenes. ${ }^{14}$ Additionally, keto functionalities present in the $\beta$-ketoenamine core could help to enhance the lifetime of the excited triplet state. $^{12,15}$ Moreover, due to the irreversible nature of the $\beta$ ketoenamine formation reaction, the novel triazine functionalized hybrid COF can offer high chemical stability even upon irradiation of light. ${ }^{13 f, g}$

The COF (TpTt) was constructed by performing reaction between melamine/1,3,5-Triazine-2,4,6-triamine (Tt) and 2,4,6Triformylphloroglucinol (Tp) aldehyde (Figure 1a, Section S2). Previously, several polymers with triazine moiety were made in the presence of a metal catalyst and higher temperatures. ${ }^{16}$ Therefore, finding a suitable method for synthesizing a crystalline porous $\mathrm{TpTt}$ COF from a melamine building block without using a metal catalyst was very challenging due to its poor reactivity. After several trials with different solvent combinations, we have produced a moderately crystalline $\mathrm{TpTt} \mathrm{COF}$ using the DMAc:DMSO (2:1) solvent combination. The PXRD patterns of TpTt display two main characteristic peaks at $2 \theta=9.7^{\circ}$ and $27.4^{\circ}$, which correspond to the reflections from the 100 and 002 planes respectively (Figure 1b). From the d spacing of the 002 peaks, we have calculated the interlayer $\pi-\pi$ stacking distance between the individual COF layers to be $3.5 \AA$ (Figure 1c). To find out a 

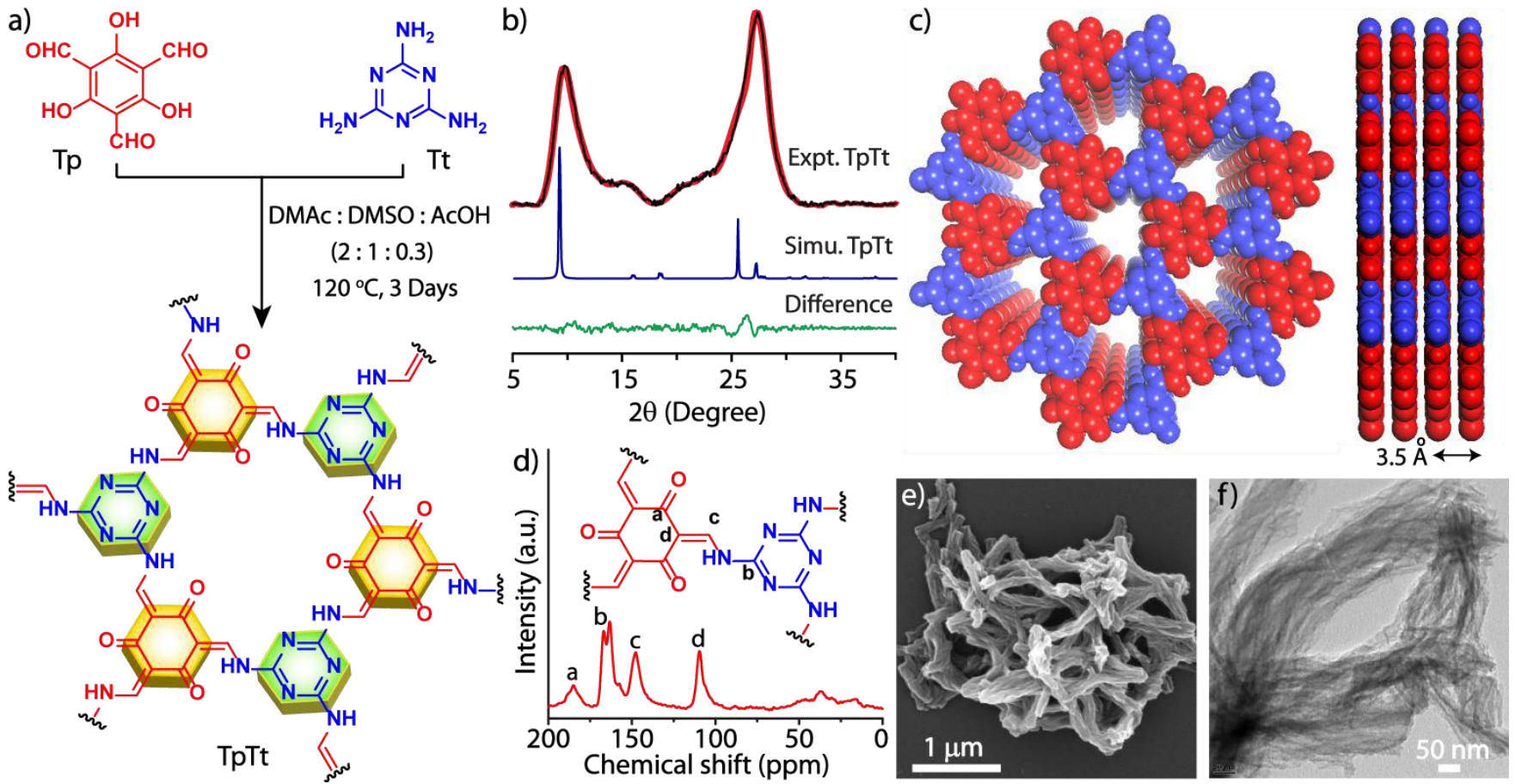

Figure 1. (a) Schematic representation of the TpTt synthesis. (b) Comparison of the experimental PXRD patterns of TpTt (black) with the simulated (blue) simulated after Pawley refinement (red), and difference plot (green). (c) Space-filling AA eclipsed stacking models of the TpTt along c and a-axis direction. (d) ${ }^{13} \mathrm{C}$ CP-MAS solid-state NMR and (e) SEM and (f) TEM images of TpTt.

reasonably fitting modeled structure of $\mathrm{TpTt}$, several stacking possibilities such as AA eclipsed, AB staggered and AA inclined models were constructed. From the Pawley refinement studies, we have observed that the simulated PXRD patterns of the AA eclipsed and AA inclined structures have a decent agreement with the experimental PXRD pattern (Section S3, Table S1). The successful incorporation of $\beta$-ketoenamine links in TpTt is indicated by the appearance of intense peaks at $1620 \mathrm{~cm}^{-1}(-\mathrm{C}=\mathrm{O}), 1523 \mathrm{~cm}^{-}$ ${ }^{1}(-\mathrm{C}=\mathrm{C})$ and $1236 \mathrm{~cm}^{-1}(-\mathrm{C}-\mathrm{N})$ in the FTIR spectra (Figure S3). The ${ }^{13} \mathrm{C}$ CP-MAS spectra of TpTt displays characteristic signals of the carbonyl carbon $(-\mathrm{C}=\mathrm{O})$ of the $\beta$-ketoenamine core at 184.9 $\mathrm{ppm}$ and the doublet peaks at $166.8 \mathrm{ppm}$ and $163.2 \mathrm{ppm}$ for aromatic carbons of the triazine core. ${ }^{17}$ The rest of the peaks corresponding to the $\mathrm{sp}^{2}$ carbons appear in the range of $147.7 \mathrm{ppm}$ to 109.5 ppm (Figure 1d, S4). SEM (Figure 1e, S9) and TEM (Figure 1f, S10) images reveal that TpTt crystallites possess fibrillar morphology. Thermogravimetric analysis (TGA) under $\mathrm{N}_{2}$ atmosphere shows the thermal stability of TpTt up to $200{ }^{\circ} \mathrm{C}$ (Figure S7).

The $\mathrm{N}_{2}$ adsorption isotherm of TpTt COF displays a type-I reversible isotherm with $277 \mathrm{~m}^{2} \mathrm{~g}^{-1}$ accessible surface area calculated by using the Brunauer-Emmett-Teller (BET) method. Furthermore, nonlocal density functional theory (NLDFT) calculations reveal that $\mathrm{TpTt}$ shows a narrow pore size distribution, with a peak maxima at $1.3 \mathrm{~nm}$ (Section S6, S7). The pore size distribution of TpTt and the length of the trans-stilbene indicate that the maximum reaction happens on the surface of the crystallites. It is experimentally observed that the porosity and the crystallinity in the framework structure (TpTt) play an important role during the photo-organocatalytic $E-Z$ isomerization process and exhibit better catalytic performance compared to similar amorphous polymer (TpTt-Poly) (Section S19). The solid-state UV-Vis diffuse reflectance spectrum of the TpTt powder shows broad absorption spectra in the visible region, having a two peak maximum at $350 \mathrm{~nm}$ and $525 \mathrm{~nm}$.
In comparison to the starting materials $\mathrm{Tp}(254$ and $340 \mathrm{~nm}$ ) and $\mathrm{Tt}(250 \mathrm{~nm})$, the absorption maxima of TpTt appears at a higher wavelength in the visible range due to extended conjugation (Figure 2a). The optical band gap of TpTt is calculated as $2.74 \mathrm{eV}$ from the UV-Vis spectra by using the Tauc plot (Figure S8). Also, we have calculated (using DFTB) the energy difference between the HOMO and LUMO orbitals of TpTt COF in several stacking modes. Among them, the theoretical energy difference between the HOMO and LUMO of TpTt in the AA slip mode is in good agreement with the calculated energy difference from experiment (Figure 3b, S17, Table S6, S7). The visible light absorption capacity, together with the ideal band gap, makes TpTt an ideal catalyst for the $E$ to $Z$ photo-isomerization reaction.

After observing the significant visible light absorbing ability of the photocatalyst, the activated TpTt catalyst is directly utilized for the trans to cis isomerization reaction by choosing transstilbene as the benchmark substrate for the optimization process. When the trans-stilbene $(0.5 \mathrm{mmol})$ in $5 \mathrm{~mL} \mathrm{DMF}$ solvent in the presence of a catalytic amount of TpTt COF $(4 \mathrm{mg})$ was irradiated using blue LEDs at room temperature, we have observed the formation of cis-stilbene (GC analysis). A 20\% yield of cisstilbene is observed after the initial 2 hours of reaction time. The yield of the cis-stilbene increased steadily with reaction time and obtained the maximum yield (90\%) at an exposure time of 18 hours (Figure 2b). Later, we have screened the effect of various solvents and observed that DMF is the optimal solvent for this transformation. It gave $90 \%$ yield of the cis-stilbene under standard reaction condition (Table 1, entry 1 , Table S2). As the amount of catalyst and solvent increase, the formation of the product also increases and $4 \mathrm{mg}$ TpTt catalyst and $5 \mathrm{~mL}$ DMF solvent are optimal for this transformation (Table S3, S4). Under similar photocatalytic conditions, melamine (Tt) yielded cisstilbene with a trace amount (Table 1, entry 3). To understand the necessity of the COF catalyst in this isomerization reaction, we have conducted a blank photocatalytic experiment without using $\mathrm{TpTt}$ in the presence of visible light (Table 1, entry 2). No peak is observed in the GC corresponding to cis-stilbene, which signifies 

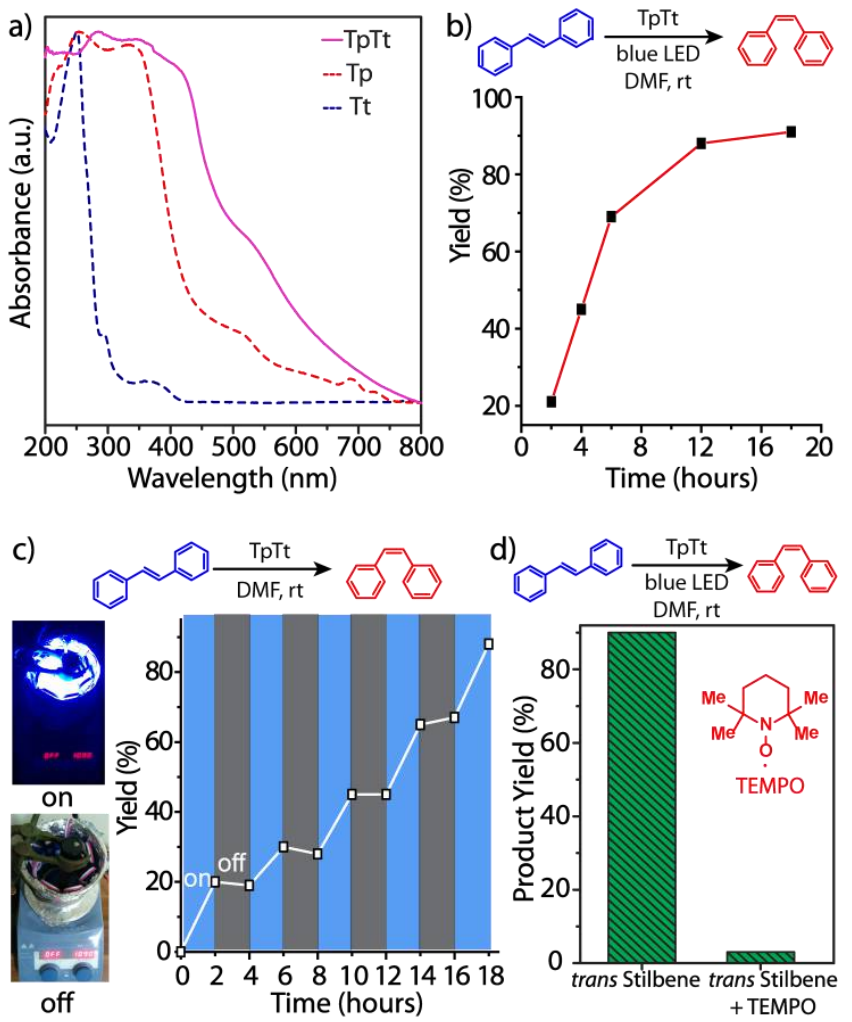

Figure 2. (a) Solid state UV-Vis diffuse reflectance spectra of TpTt (pink), Tp (red) and Tt (blue). (b) The photocatalytic yield of cis-stilbene at different time intervals. (c) Light on off experiment study over time. (d) The photocatalytic yield of cisstilbene in the absence and presence of a free radical scavenger (R.S.) TEMPO.

that in the absence of the catalyst, only higher energy UV radiation is necessary for the trans to cis isomerization process. To prove that the reaction is neither thermodynamically nor kinetically controlled, we have performed the reactions at $80^{\circ} \mathrm{C}$

Table 1. Optimization of the Reaction Conditions. ${ }^{a}$

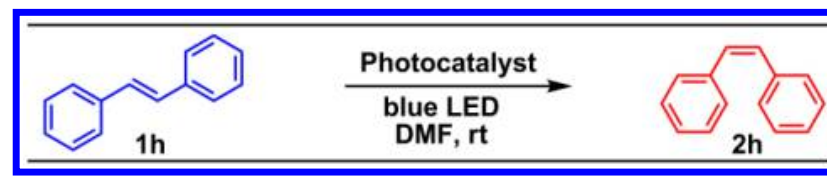

$\begin{array}{ccccc}\text { entry } & \text { catalyst } & \text { temp } & \text { solvent } & \text { yield[\% }]^{b} \\ 1 & \mathrm{TpTt} & \mathrm{rt} & \mathrm{DMF} & 90 \\ 2 & - & \mathrm{rt} & \mathrm{DMF} & \text { trace } \\ 3 & \text { Melamine (Tt) } & \mathrm{rt} & \mathrm{DMF} & \text { trace } \\ 4^{[\mathrm{cc}]} & \mathrm{TpTt}+\mathrm{TEMPO} & \mathrm{rt} & \mathrm{DMF} & \text { trace } \\ 5^{[\mathrm{d}]} & \mathrm{TpTt} & 80^{\circ} \mathrm{C} & \mathrm{DMF} & - \\ 6^{[\mathrm{d}]} & \mathrm{TpTt} & \mathrm{rt} & \mathrm{DMF} & -\end{array}$

${ }^{a}$ All reactions were conducted with $0.5 \mathrm{mmol}$ of trans-stilbene (1 equiv.), $4 \mathrm{mg} \mathrm{TpTt}$ catalyst, in $5 \mathrm{~mL}$ solvents at $\mathrm{rt}$ under blue LEDs irradiation (total $36 \mathrm{~W}$; each LED has $1 \mathrm{~W}$ power) for $18 \mathrm{~h}$. ${ }^{b}$ Based on GC analysis using n-decane as an internal standard. ${ }^{c} 4$ equiv. TEMPO was added into the reaction mixture. ${ }^{d}$ In absence of blue LEDs source.
(Table 1, entry 5) and rt (Table 1, entry 6) in absence of blue LED light. No product ( $c i s$-stilbene) is formed under these conditions. TpTt has shown good yield towards the trans to cis photoisomerization of different stilbene substrates (Table 2). The significance of visible light source is essential for this transformation was investigated by performing the light on-off experiment over time (Figure 2c). The reaction proceeds when the light is on, and the conversion stops when the light is turned off, which indicates that the reaction happens through a photocatalytic pathway. To check the recyclability of the TpTt catalyst, we have carried out the photocatalytic activity test up to four cycles. Even after four cycles, the TpTt catalyst showed similar photocatalytic activity (Section S15). The unchanged FTIR, PXRD, TEM and gas adsorption isotherm of TpTt catalyst after 20 hours of photo exposure in DMF suggests the high photostability of the catalyst (Section S17).

Table 2. Porous COF Catalyzed Photoisomerization of Alkene Substrates Scope. ${ }^{a, b}$

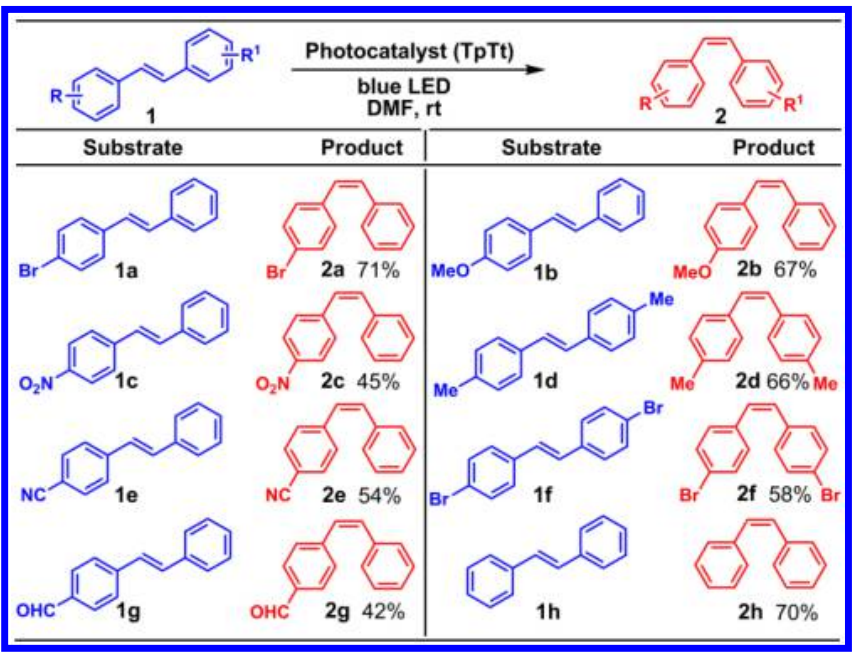

${ }^{a}$ All the reaction were conducted with $0.5 \mathrm{mmol}$ of trans-stilbene

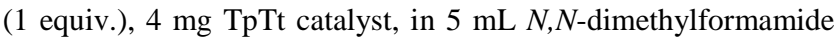
at rt under blue LEDs irradiation (total $36 \mathrm{~W}$; each LED has $1 \mathrm{~W}$ power) for $18 \mathrm{~h} .{ }^{b}$ Isolated yields.

To explore the catalytic reaction mechanism, we have performed a controlled photocatalytic reaction using a radical scavenger (R.S.) TEMPO. In the presence of 4 eq TEMPO R.S. in the reaction medium, the yield of the cis product decreases significantly [ $3 \%]$ under the optimized conditions, which confirms that the reaction proceeds through a biradical intermediate state (Figure 2d, Table 1, entry 4). ${ }^{10 b-c, 11 c, 18}$ To get further insight about the reaction mechanism, we have performed theoretical calculations of energy states of different possible reaction intermediates during the isomerization reaction (Section S18). The results indicate that the first $\mathrm{TpTt}$ absorbs visible light and gets excited from the ground state to the first singlet excited state $\left(S_{0}\right.$ to $\left.S_{1}\right)$. After the intersystem crossing (ISC) TpTt reaches the energetically more stable triplet excited state $\left(\mathrm{T}_{1}\right)$ and subsequently interacts with trans-stilbene and transfers its energy ${ }^{19}$ to the trans-stilbene. This energy transfer helps trans-stilbene to convert itself into its biradical triplet intermediate state $\left(\mathrm{T}_{1}\right)$. This triplet intermediate state then gets converted to the product cis-stilbene (Figure 3a).

In conclusion, for the first time, we could successfully develop a heterogeneous TpTt photocatalyst for the visible light-induced isomerization reaction of trans to cis-stilbene. The hybrid $\mathrm{TpTt}$ photocatalyst is synthesized from two types of distinct photoactive building blocks. Due to the $\beta$-ketoenamine linked 


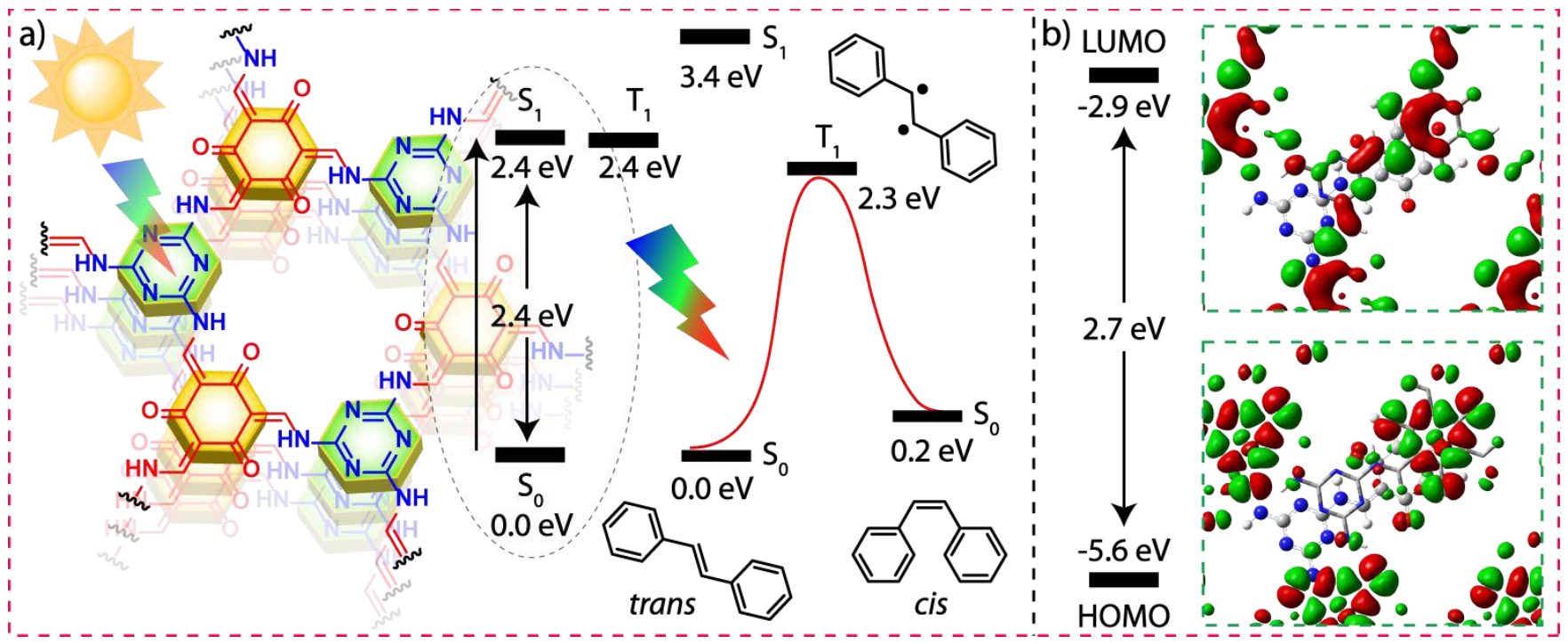

Figure 3. (a) Mechanistic representation of trans to cis photoisomerization of stilbene using the TpTt COF catalyst. (b) Pictorial representation of HOMO and LUMO orbitals and their energy levels for the TpTt COF catalyst. The energy levels of the orbitals are not exactly to scale.

structure, the novel TpTt catalyst displays high chemical stability even upon irradiation of light and broad substrates scope and retains its photocatalytic activity even after four consecutive reaction cycles. We believe that the novel COF photocatalyst will be a promising candidate for the scalable and cost-effective synthesis of industrially important $c$ is olefins.

\section{ASSOCIATED CONTENT}

\section{Supporting Information}

Experimental procedures and characterization data. This material is available free of charge via the Internet at http://pubs.acs.org.

\section{AUTHOR INFORMATION}

\section{Corresponding Author}

r.banerjee@ncl.res.in

eb.raman@ncl.res.in

\section{Notes}

The authors declare no competing financial interests.

\section{ACKNOWLEDGMENT}

M.B., S.K. and M.K.S. acknowledge CSIR India. R.B. acknowledges IISER-Kolkata startup grant and DST-Swarna Jayanti Fellowship grant (DST/SJF/CSA-02/2016-2017) for funding. E.B. acknowledges SERB (EMR/2015/000030) for finantial support. M.A.A. acknowledges HPC time on THOMAS through the Materials Chemistry Consortium (EP/L000202). We acknowledge S. Karak for his helpful suggestions and Dr. T. G. Ajithkumar for the NMR facility.

\section{REFERENCES}

(1) (a) Boëdec, A.; Sicard, H.; Dessolin, J.; Herbette, G.; Ingoure, S.; Raymond, C.; Belman, C.; Kraus, J. -L. Synthesis and Biological Activity of Phosphonate Analogues and Geometric Isomers of the Highly Potent Phosphoantigen (E)-1-Hydroxy-2-methylbut-2-enyl 4-Diphosphate. $J$. Med. Chem. 2008, 51, 1747-1754. (b) Chhabra, N.; Aseri, M. L.; Padmanabhan, D. A review of drug isomerism and its significance. Int. J. Appl. Basic Med. Res. 2013, 3, 16-18. (c) Williams, M. L.; Lennard, M. S.; Martin, I. J.; Tucker, G. T. Interindividual variation in the isomerization of
4- hydroxytamoxifen by human liver microsomes: involvement of cytochromes P450. Carcinogenesis 1994, 15, 2733-2738. (d) McCalley, A.; Kaja, S.; Payne, A.; Koulen, P. Resveratrol and Calcium Signaling: Molecular Mechanisms and Clinical Relevance. Molecules 2014, 19, 73277340. (e) Frazzi, R.; Tigano, M. The Multiple Mechanisms of Cell Death Triggered by Resveratrol in Lymphoma and Leukemia. Int. J. Mol. Sci. 2014, 15, 4977-4993.

(2) Brown, J. A.; Goldblum, B. L.; Bernstein, L. A.; Bleuel, D. L.; Brickner, N. M.; Caggiano, J. A.; Daub, B. H.; Kaufman, G. S.; Hatarik, R.; Phillips, T. W.; Wender, S. A.; Bibber, K. van.; Vujic, J.; Zaitseva, N. P. Relative light yield and temporal response of a stilbene-doped bibenzyl organic scintillator for neutron detection. J. Appl. Phvs. 2014, 115, 193504-193508.

(3) Hara, M.; Samori, S.; Cai, X.; Fujitsuka, M.; Majima, T. Importance of Properties of the Lowest and Higher Singlet Excited States on the Resonant Two-Photon Ionization of Stilbene and Substituted Stilbenes Using Two-Color Two-Lasers. J. Phvs. Chem. A 2005, 109, 9831-9835.

(4) Song, D. H.; Yoo, H. Y.; Kim, J. P. Synthesis of stilbene-based azo dyes and application for dichroic materials in poly(vinyl alcohol) polarizing films. Dves Pigm. 2007, 75, 727-731.

(5) (a) Gai, F.; Hasson, K. C.; MacDonald, J. C.; Anfinrud, P. A. Chemical Dynamics in Proteins: The Photoisomerization of Retinal in Bacteriorhodopsin. Science 1998, 279, 1886-1891. (b) Cresswell, A. J.; Eey, S. T.-C.; Denmark, S. Catalytic, stereospecific syn-dichlorination of alkenes. Nat. Chem. 2015, 7, 146-152. (c) Metternich, J. B.; Artiukhin, D. G.; Holland, M. C.; von Bremen-Kuhne, M.; Neugebauer, J.; Gilmour, R. Photocatalytic $\mathrm{E} \rightarrow \mathrm{Z}$ Isomerization of Polarized Alkenes Inspired by the Visual Cycle: Mechanistic Dichotomy and Origin of Selectivity. J. Org. Chem. 2017, 82, 9955-9977.

(6) Siau, W. -Y.; Zhang, Y.; Zhao, Y. Stereoselective Synthesis of ZAlkenes. Top. Curr. Chem. 2012, 327, 33-58.

(7) (a) Staden, L. F. V.; Gravestock, D.; Ager, D. J. New developments in the Peterson olefination reaction. Chem. Soc. Rev. 2002, 31, 195-200. (b) Negishi, E.-I.; Huang, Z.; Wang, G.; Mohan, S.; Wang, C.; Hattori, H. Recent Advances in Efficient and Selective Synthesis of Di-, Tri-, and Tetrasubstituted Alkenes via Pd-Catalyzed Alkenylation-Carbonyl Olefination Synergy. Acc. Chem. Res. 2008, 41, 1474-1485. (c) Huang, Z.; Negishi, E.-I. Highly Stereo- and Regioselective Synthesis of (Z)Trisubstituted Alkenes via 1-Bromo-1-alkyne Hydroboration-Migratory Insertion-Zn-Promoted Iodinolysis and Pd-Catalyzed Organozinc CrossCoupling. J. Am. Chem. Soc. 2007, 129, 14788-14792. (d) Meek, S. J.; O’Brien, R. V.; Llaveria, J.; Schrock, R. R.; Hoveyda, A. H. Catalytic Zselective olefin cross-metathesis for natural product synthesis. Nature 2011, 471, 461-466. (e) Koh, M. J.; Khan, R. K. M.; Torker, S.; Hoveyda, A. H. Broadly Applicable Z- and Diastereoselective Ring-Opening/CrossMetathesis Catalyzed by a Dithiolate Ru Complex Angew. Chem. Int. Ed. 
2014, 53, 1968-1972. (f) Endo, K.; Grubbs, R. H. Chelated Ruthenium Catalysts for Z-Selective Olefin Metathesis. J. Am. Chem. Soc. 2011, 133, 8525-8527.

(8) (a) Hoffmann, N. Photochemical Reactions as Key Steps in Organic Synthesis. Chem. Rev. 2008, 108, 1052-1103. (b) Waldeck, D. H. Photoisomerization dynamics of stilbenes. Chem. Rev. 1991, 91, 415-436. (c) Arai, T.; Tokumaru, K. Photochemical one-way adiabatic isomerization of aromatic olefins. Chem. Rev. 1993, 93, 23-39. (d) Prier, C. K.; Rankic, D. A.; MacMillan, D. W. C. Visible Light Photoredox Catalysis with Transition Metal Complexes: Applications in Organic Synthesis. Chem. Rev. 2013, 113, 5322-5363.

(9) (a) Happ, B.; Winter, A.; Hager, M. D.; Schubert, U. S. Photogenerated avenues in macromolecules containing $\mathrm{Re}(\mathrm{I}), \mathrm{Ru}(\mathrm{II}), \mathrm{Os}(\mathrm{II})$, and Ir(III) metal complexes of pyridine-based ligands. Chem. Soc. Rev. 2012 41, 2222-2255. (b) Meyer, T. J. Chemical approaches to artificial photosynthesis. Acc. Chem. Res. 1989, 22, 163-170.

(10) (a) Zhao, J.; Wu, W.; Sun, J.; Guo, S. Triplet photosensitizers: from molecular design to applications. Chem. Soc. Rev. 2013, 42, 5323-5351. (b) Fabry, D. C.; Ronge, M. A.; Rueping, M. Immobilization and Continuous Recycling of Photoredox Catalysts in Ionic Liquids for Applications in Batch Reactions and Flow Systems: Catalytic Alkene Isomerization by Using Visible Light. Chem. - Eur. J. 2015, 21, 5350-5354. (c) Singh, K.; Staig, S. J.; Weaver, J. D. Facile Synthesis of Z-Alkenes via Uphill Catalysis. J. Am. Chem. Soc. 2014, 136, 5275-5278.

(11) (a) Ravelli, D.; Fagnoni, M.; Albini, A. Photoorganocatalysis. What for? Chem. Soc. Rev. 2013, 42, 97-113. (b) Metternich, J. B.; Gilmour, R. A Bio-Inspired, Catalytic $\mathrm{E} \rightarrow \mathrm{Z}$ Isomerization of Activated Olefins.. Am. Chem. Soc. 2015, 137, 11254-11257. (c) Cai, W.; Fan, H.; Ding, D.; Zhang, Y.; Wang, W. Synthesis of Z-alkenes via visible light promoted photocatalytic $\mathrm{E} \rightarrow \mathrm{Z}$ isomerization under metal-free conditions. Chem. Commun. 2017, 53, 12918-12921.

(12) (a) Zhao, Y. -P.; Yang, L. -Y.; Liu, R. S. H. Designing systems for one-way trans to cis photoisomerization for solar reactions. Green Chem. 2009, 11, 837-842. (b) Herkstroeter, W. G.; Hammond, G. S. Mechanisms of Photochemical Reactions in Solution. XXXIX. ${ }^{1}$ Study of Energy Transfer by Kinetic Spectrophotometry. J. Am. Chem. Soc. 1966, 88, 47694777. (c) Liu, R. S. H.; Turro, N. J.; Hammond, G. S. Mechanisms of Photochemical Reactions in Solution. XXXI. Activation and Deactivation of Conjugated Dienes by Energy Transfer. J. Am. Chem. Soc. 1965, 87, 3406-3412.

(13) (a) Côté, A. P.; Benin, A. I.; Ockwig, N. W.; O’Keeffe, M.; Matzger, A. J.; Yaghi, O. M. Porous, Crystalline, Covalent Organic Frameworks. Science 2005, 310, 1166-1170. (b) Colson, J. W.; Dichtel, W. R. Rationally synthesized two-dimensional polymers. Nat. Chem. 2013, 5, 453-465. (c) Dinga, S.-Y.; Wang, W. Covalent organic frameworks (COFs): from design to applications. Chem. Soc. Rev. 2013, 42, 548-568. (d) Calik, M.; Sick, T.; Dogru, M.; Döblinger, M.; Datz, S.; Budde, H.; Hartschuh, A.; Auras, F.; Bein, T. From Highly Crystalline to Outer SurfaceFunctionalized Covalent Organic Frameworks-A Modulation Approach. J. Am. Chem. Soc. 2016, 138, 1234-1239. (e) Pachfule, P.; Acharjya, A.; Roeser, J.; Langenhahn, T.; Schwarze, M.; Schomäcker, R.; Thomas, A.; Schmidt, J. Diacetylene Functionalized Covalent Organic Framework (COF) for Photocatalytic Hydrogen Generation. J. Am. Chem. Soc. 2018, 140, 1423-1427. (f) Kandambeth, S.; Mallick, A.; Lukose, B.; Mane, M. V.; Heine, T.; Banerjee, R. Construction of Crystalline 2D Covalent Organic Frameworks with Remarkable Chemical (Acid/Base) Stability via a Combined Reversible and Irreversible Route. J. Am. Chem. Soc. 2012, 134, 19524-19527. (g) Shinde, D. B.; Aiyappa, H. B.; Bhadra, M.; Biswal, B. P.; Wadge, P.; Kandambeth, S.; Garai, B.; Kundu, T.; Kurungot, S.; Banerjee, R. A mechanochemically synthesized covalent organic framework as a proton-conducting solid electrolyte. J. Mater. Chem. A 2016, 4, 2682-2690. (h) Ding, S.-Y.; Gao, J.; Wang, Q.; Zhang, Y.; Song, W.-G.; Su, C.-Y.; Wang, W. Construction of Covalent Organic Framework for Catalysis: Pd/COF-LZU1 in Suzuki-Miyaura Coupling Reaction. J. Am. Chem. Soc. 2011, 133, 19816-19822. (i) Bhadra, M.; Sasmal, H. S.; Basu, A.; Midya, S. P.; Kandambeth, S.; Pachfule, P.; Balaraman, E.; Banerjee, R. Predesigned Metal-Anchored Building Block for In Situ Generation of Pd Nanoparticles in Porous Covalent Organic Framework: Application in Heterogeneous Tandem Catalysis. ACS Appl. Mater. Interfaces 2017, 9, 13785-13792. (j) Vyas, V. S.; Haase, F.; Stegbauer, L.; Savasci, G.; Podjaski, F.; Ochsenfeld, C.; Lotsch, B. V. A tunable azine covalent organic framework platform for visible light-induced hydrogen generation. Nat. Commun. 2015, 6, 8508-8516. (k) Fang, Q.; Gu, S.; Zheng, J.; Zhuang, Z.;
Qiu, S.; Yan, Y. 3D Microporous Base-Functionalized Covalent Organic Frameworks for Size-Selective Catalysis. Angew. Chem. Int. Ed. 2014, 53, 2878-2882. (1) Wang, X.; Han, X.; Zhang, J.; Wu, X.; Liu, Y.; Cui, Y. Homochiral 2D Porous Covalent Organic Frameworks for Heterogeneous Asymmetric Catalysis. J. Am. Chem. Soc. 2016, 138, 12332-12335. (m) Han, X.; Huang, J.; Yuan, C.; Liu, Y.; Cui, Y. Chiral 3D Covalent Organic Frameworks for High Performance Liquid Chromatographic Enantioseparation. J. Am. Chem. Soc. 2018, 140, 892-895. (n) Sun, Q.; Aguila, B.; Perman, J.; Nguyen, N.; Ma, S. Flexibility Matters: Cooperative Active Sites in Covalent Organic Framework and Threaded Ionic Polymer. $\mathrm{J}$. Am. Chem. Soc. 2016, 138, 15790-15796. (o) Xu, H.; Gao, J.; Jiang, D. Stable, crystalline, porous, covalent organic frameworks as a platform for chiral organocatalysts. Nat. Chem. 2015, 7, 905-912. (p) Liu, W.; Cao, Y.; Wang, W.; Gong, D.; Cao, T.; Qian, J.; Iqbal, K.; Qin, W.; Guo, H. Mechanochromic luminescent covalent organic frameworks for highly selective hydroxyl radical detection. Chem. Commun. 2019, 55, 167-170. **

(14) Ohara, K.; Inokuma, Y.; Fujita, M. The Catalytic Z to E Isomerization of Stilbenes in a Photosensitizing Porous Coordination Network. Angew. Chem. Int. Ed. 2010, 49, 5507-5509.

(15) Hammond, G. S.; Saltiel, J.; Lamola, A. A.; Turro, N. J.; Bradshaw, J. S.; Cowan, D. O.; Counsell, R. C.; Vogt, V.; Dalton, C. Mechanisms of Photochemical Reactions in Solution. XXII. ${ }^{1}$ Photochemical cis-trans Isomerization. J. Am. Chem. Soc. 1964, 86, 3197-3217.

(16) (a) Schwab, M. G.; Fassbender, B.; Spiess, H. W.; Thomas, A.; Feng, X.; Müllen, K. Catalyst-free Preparation of Melamine-Based Microporous Polymer Networks through Schiff Base Chemistry. J. Am. Chem. Soc. 2009, 131, 7216-7217. (b) Holst, J. R.; Gillan, E. G. From Triazines to Heptazines: Deciphering the Local Structure of Amorphous Nitrogen-Rich Carbon Nitride Materials. J. Am. Chem. Soc. 2008, 130, 7373-7379. (c) Lau, V. W.-h.; Mesch, M. B.; Duppel, V.; Blum, V.; Senker, J.; Lotsch, B. V. Low-Molecular-Weight Carbon Nitrides for Solar Hydrogen Evolution. J. Am. Chem. Soc. 2015, 137, 1064-1072. (d) Kuecken, S.; Acharjya, A.; Zhi, L.; Schwarze, M.; Schomäcker, R.; Thomas, A. Fast tuning of covalent triazine frameworks for photocatalytic hydrogen evolution. Chem. Commun. 2017, 53, 5854-5857.

(17) Jürgens, B.; Irran, E.; Senker, J.; Kroll, P.; Müller, H.; Schnick, W. Melem (2,5,8-Triamino-tri-s-triazine), an Important Intermediate during Condensation of Melamine Rings to Graphitic Carbon Nitride: Synthesis, Structure Determination by X-ray Powder Diffractometry, Solid-State NMR, and Theoretical Studies. J. Am. Chem. Soc. 2003, 125, 1028810300 .

(18) Caldwell, R. A.; Zhou, L. Are Perpendicular Alkene Triplets Just 1,2Biradicals? Studies with the Cyclopropylcarbinyl Clock. J. Am. Chem. Soc. 1994, 116, 2271-2275.

(19) Klosterman, J. K.; Iwamura, M.; Tahara, T.; Fujita, M. Energy Transfer in a Mechanically Trapped Exciplex. J. Am. Chem. Soc. 2009, 131, 9478-9479.

[**]Although the pictorial representation of both the COFs (COF-TpMA (MC) and TpTt COF) is similar but we believe that our synthesized material has higher crystallinity, porosity, purity and accuracy of the structure prediction from the experimentally observed data compared to the previous report. 
Synopsis TOC

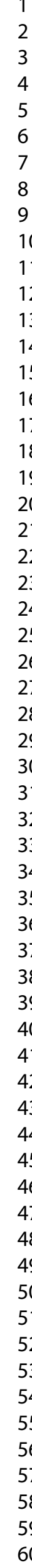

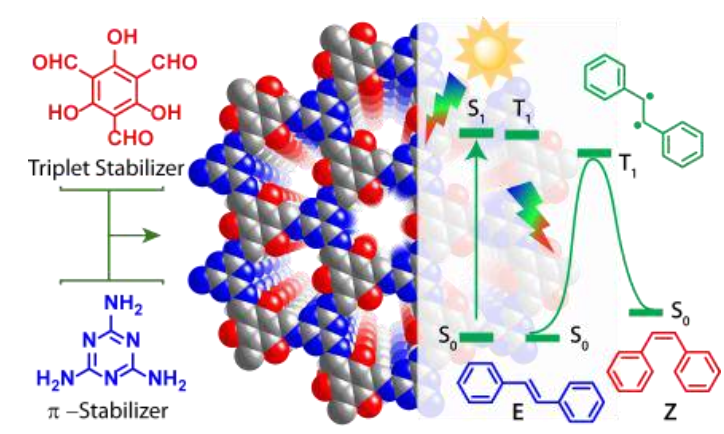




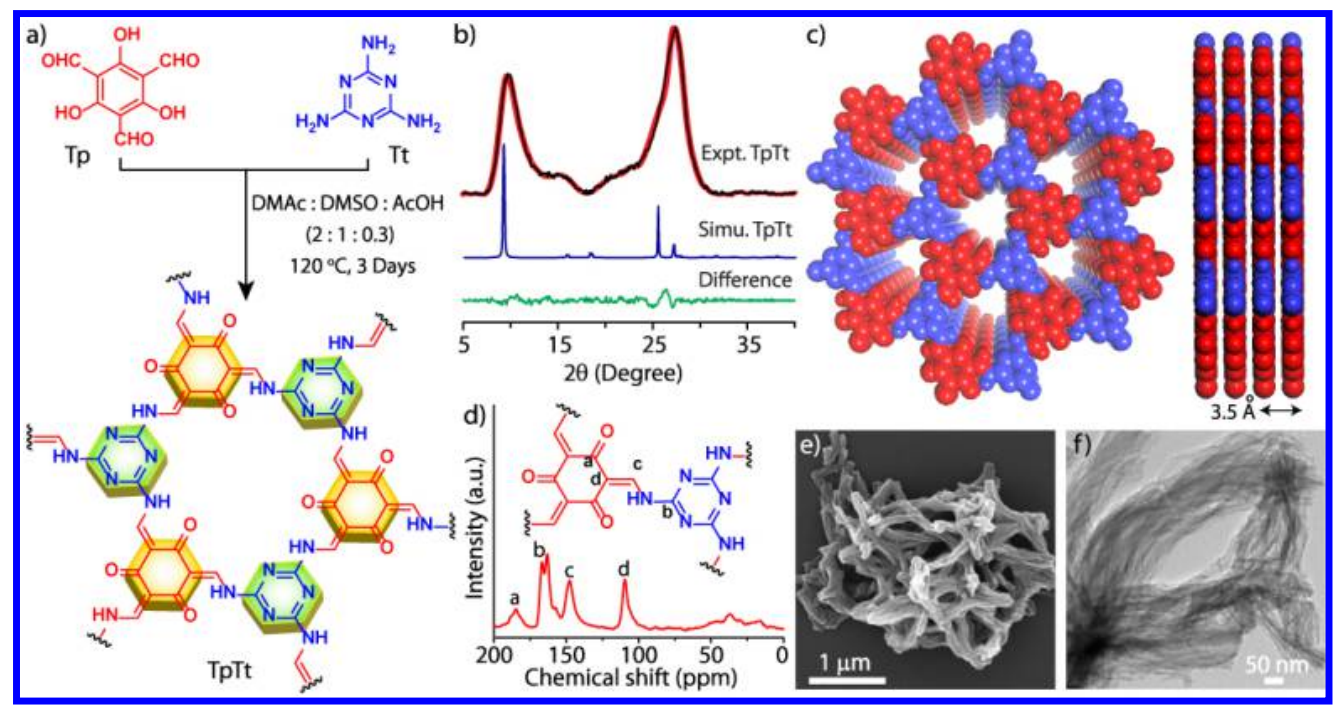

Figure 1 

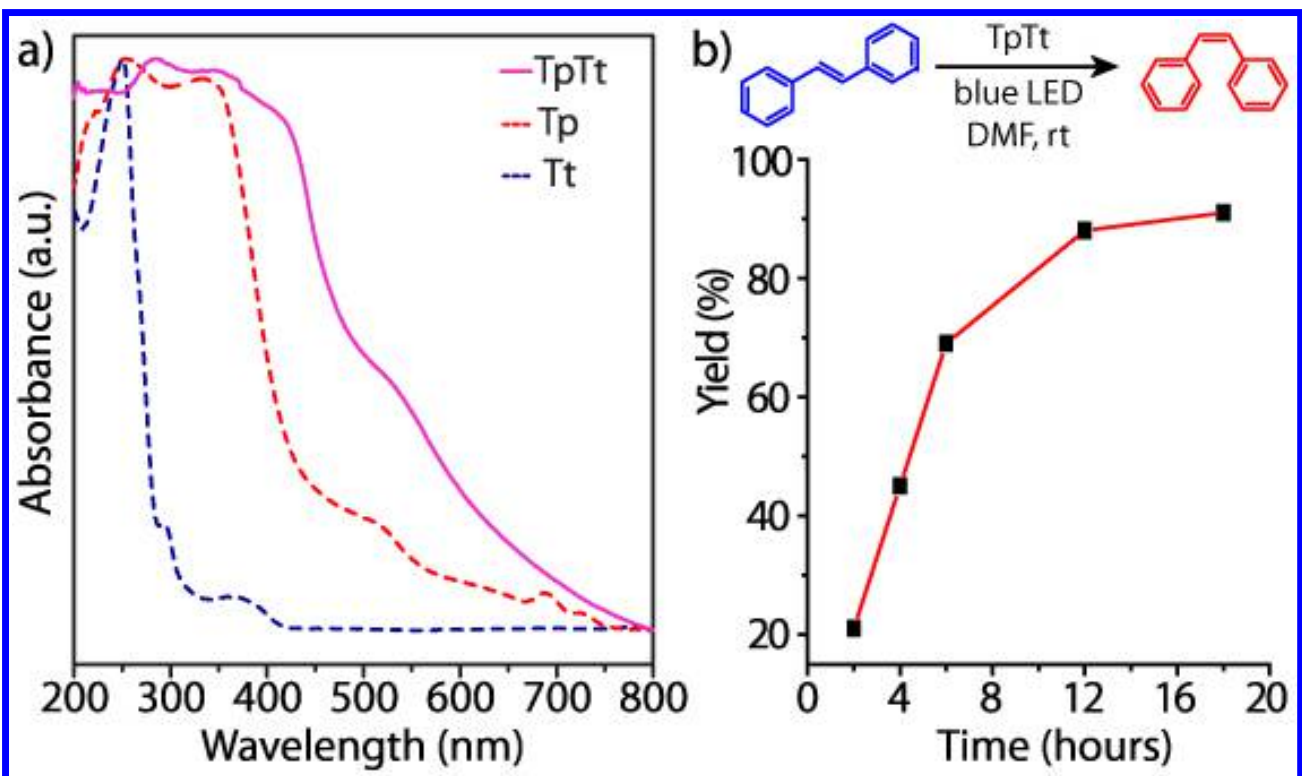

c)
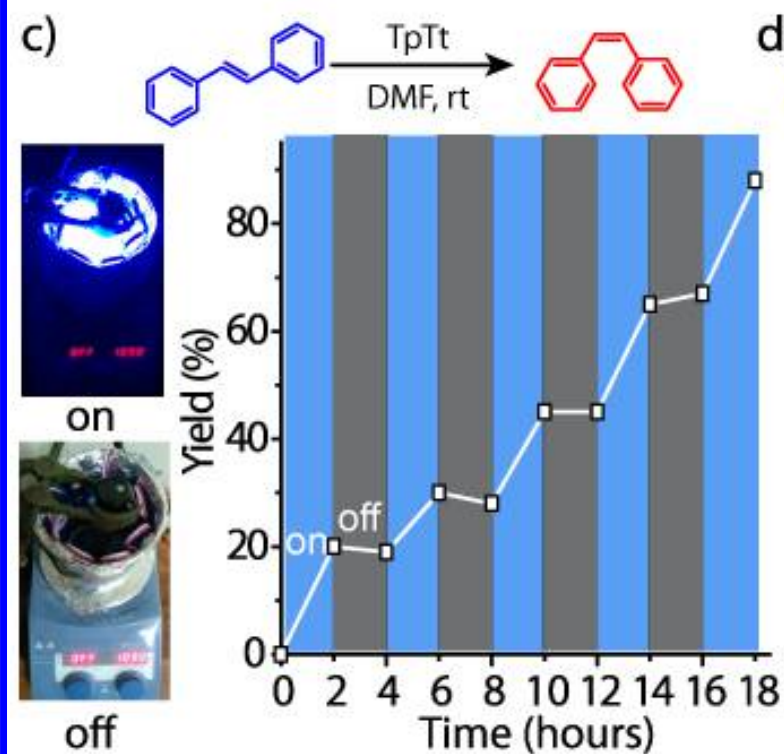

d)

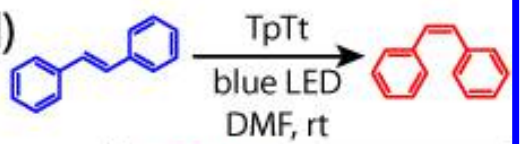

Figure 2 


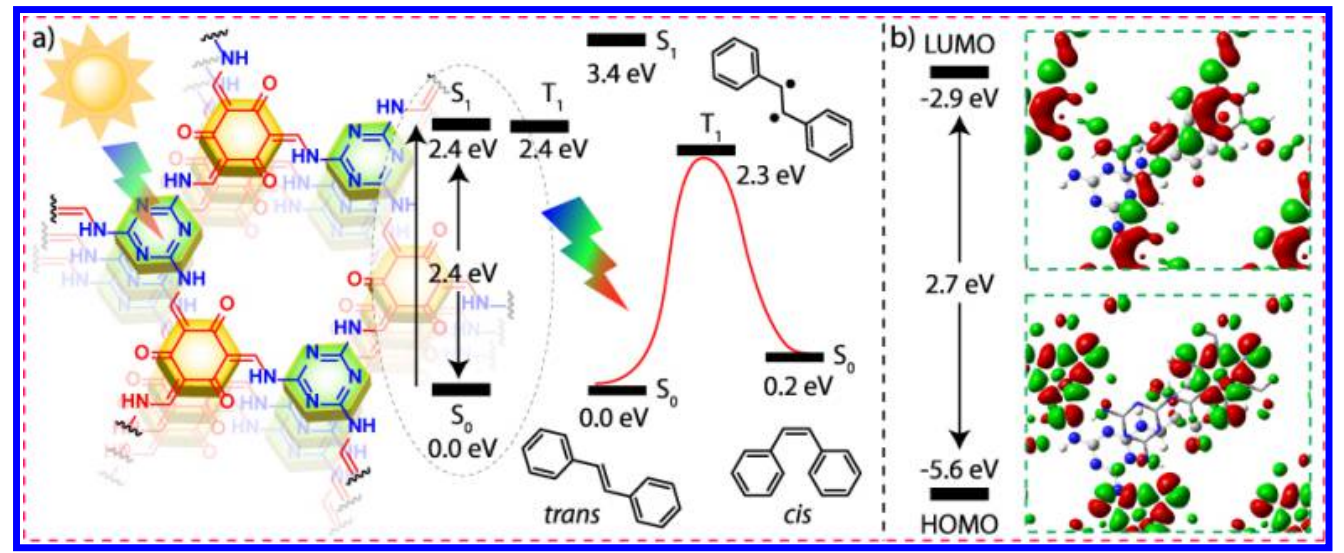

Figure 3 


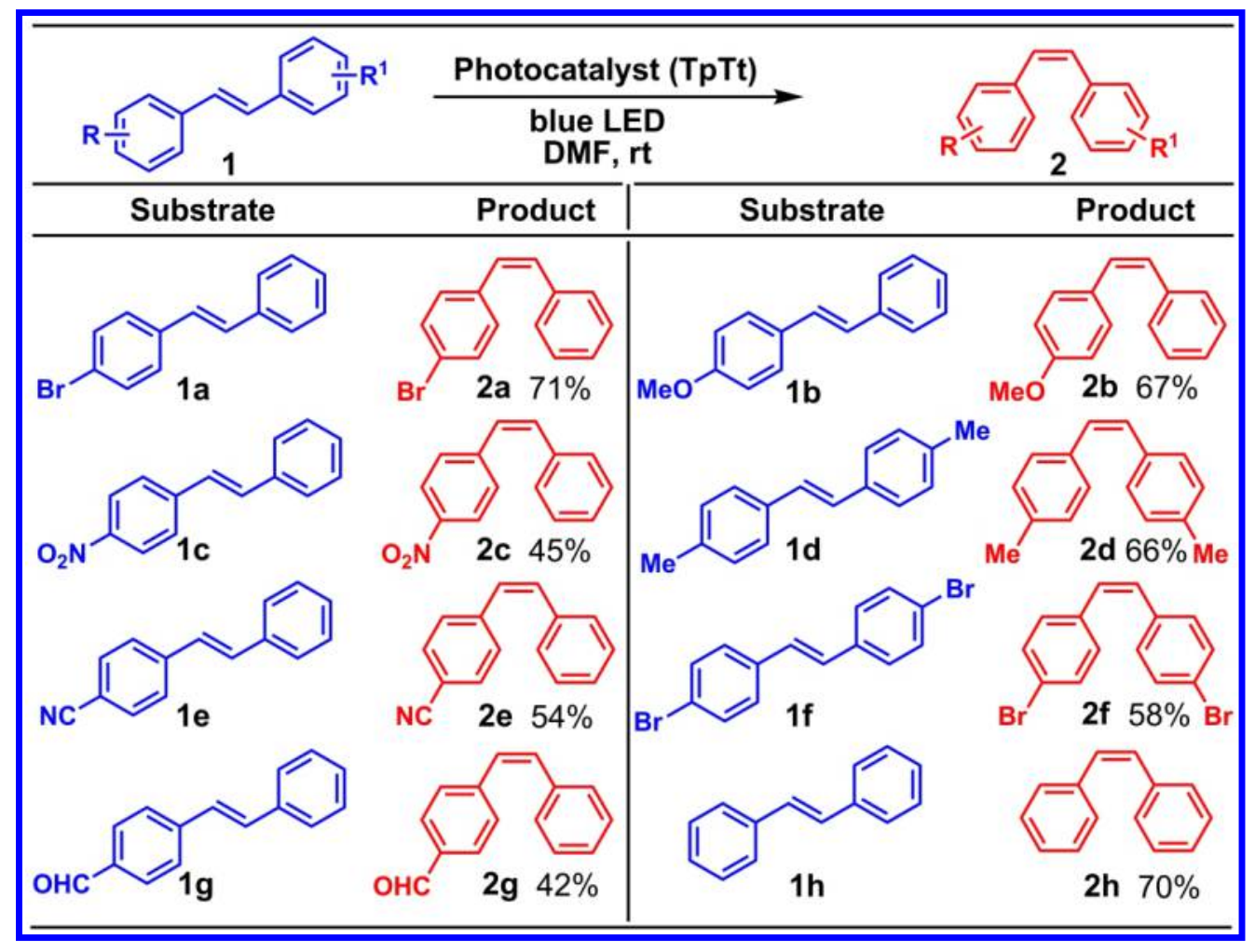

Figure_SubstrateScope

$162 \times 122 \mathrm{~mm}(600 \times 600 \mathrm{DPI})$ 


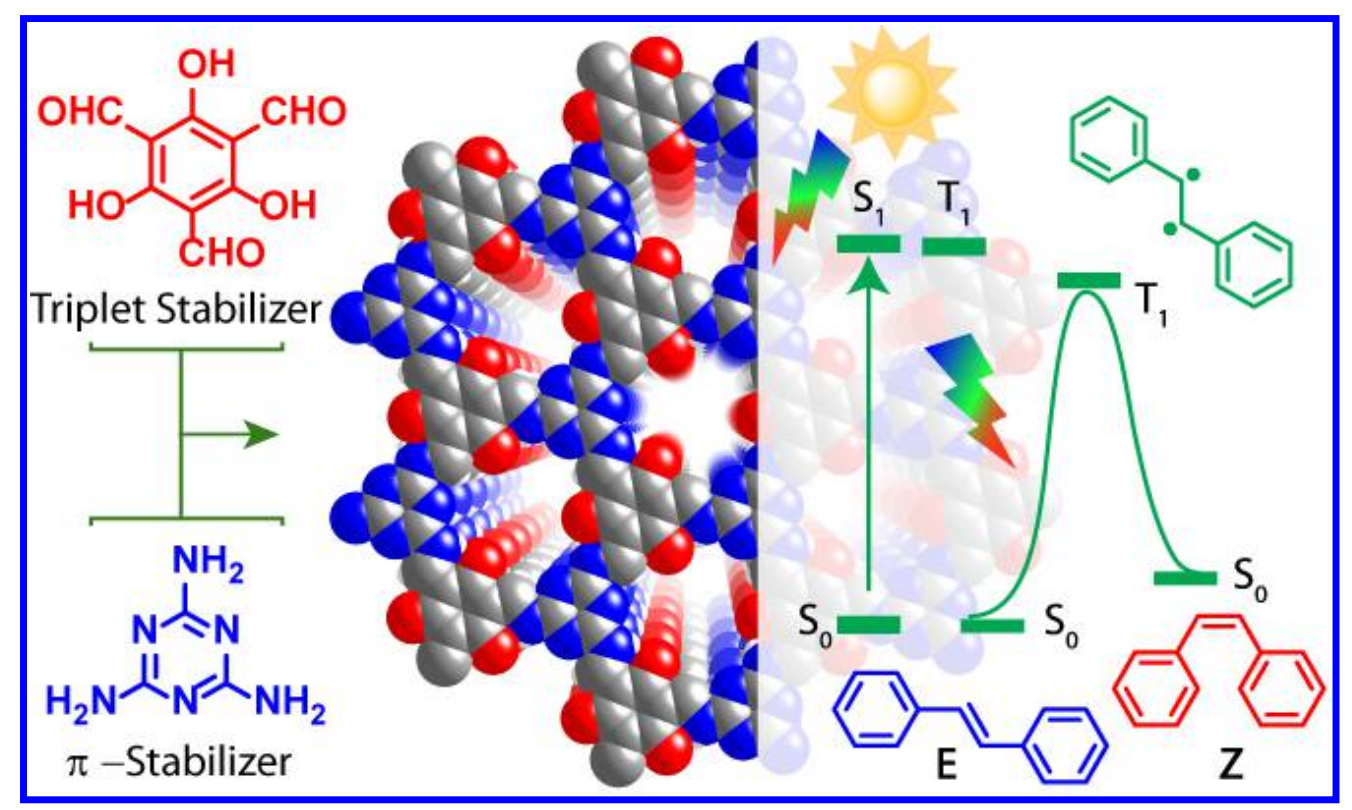

Figure TOC 